\title{
Cancer detection in excised breast tumors using terahertz imaging and spectroscopy
}

\author{
Magda El-Shenawee ${ }^{\mathrm{a}, *}$, Nagma Vohra ${ }^{\mathrm{a}}$, Tyler Bowman ${ }^{\mathrm{a}}$ and Keith Bailey ${ }^{\mathrm{b}}$ \\ ${ }^{a}$ Department of Electrical Engineering, University of Arkansas, Fayetteville, USA \\ b Oklahoma Animal Disease Diagnostic Laboratory, Oklahoma State University, Stillwater, \\ Oklahoma, USA
}

\begin{abstract}
Terahertz imaging and spectroscopy has demonstrated a potential for differentiating tissue types of excised breast cancer tumors. Pulsed terahertz technology provides a broadband frequency range from $0.1 \mathrm{THz}$ to $4 \mathrm{THz}$ for detecting cancerous tissue. Tumor tissue types of interest include cancer typically manifested as infiltrating ductal or lobular carcinomas, fibro-glandular (healthy connective tissues) and fat. In this work, images of breast tumors excised from human and animal models are reviewed. In addition to alternate fresh tissues, breast cancer tissue phantoms are developed to further evaluate terahertz imaging and the potential use of contrast agents. Terahertz results are successfully validated with pathology images, showing strong differentiation between cancerous and healthy tissues for all freshly excised tissues and types. The advantages, challenges and limitations of $\mathrm{THz}$ imaging of breast cancer are discussed.
\end{abstract}

Keywords: Breast cancer, terahertz imaging, terahertz spectroscopy, margin assessment, lumpectomy

\section{Introduction}

Since the first steps towards reliable terahertz (THz) sources nearly 20 years ago, researchers have been racing to develop hardware systems that utilize this historical "terahertz gap" from 0.1 to $10 \mathrm{THz}$ for medical and engineering applications [9]. One such application is breast cancer, representing 11.6\% of all global cancer cases with more than two million estimated new diagnoses in 2018 [8]. The two common surgical treatments for invasive breast cancer are mastectomy, or full breast removal, and lumpectomy, removal of just the tumor bulk and margin. With early diagnosis, lumpectomy is generally preferred over radical mastectomy (57\% of early detections) and has shown to be just as effective at preventing cancer spread recurrence [13]. Lumpectomy also has reduced cosmetic damage and therefore stands to become more common as early cancer screening techniques improve.

The primary surgical concern for lumpectomy procedures is the margin assessment. A positive margin denotes cancer on the outside border of the removed tumor, and therefore in the surgical cavity, while a negative margin indicates that only healthy tissue is present at the surgical edge. Since local recurrence occurs in the original excision site in $75-90 \%$ of cases, positive margins demand additional excision to ensure patient health [17]. The American Society for Radiation Oncology (ASTRO) guidelines state "Negative margins (no ink on tumor) optimize [ipsilateral breast tumor recurrence]. Wider margins widths do not significantly lower this risk" [18]. As such there is a strong need for accurate margin assessment that still maintains conservative tissue excision.

\footnotetext{
*Corresponding author. E-mail: magda@uark.edu.
} 
The golden standard for margin assessment is pathology imaging. Formalin-fixed, paraffin-embedded (FFPE) blocks of excised tumor tissue are sectioned into 4-5 $\mu \mathrm{m}$ thick slices and stained with hematoxylin and eosin (H\&E) for pathology assessment under a microscope. However, this process can take from several days to weeks, and any further excisions require a second surgery that puts additional strain on patients and medical care providers [15]. While intraoperative techniques like radio frequency ablation and cavity shaving are effective in reducing positive margin rates, these techniques are generally undirected and can cause additional unnecessary damage without a method to locate any remaining cancer [16]. With a positive margin rate of $20-40 \%$ in conventional lumpectomy procedures, this creates a critical need for a fast, intraoperative margin assessment tool [20].

$\mathrm{THz}$ technology has shown significant differentiation between various diseased and healthy tissues [12]. THz radiation is relatively low-power and non-ionizing, making it biologically safe for both in vivo and excised tissues [22]. THz frequencies have a greater imaging resolution than microwaves due to a shorter wavelength and show improved transmittance in fatty tissues over infrared techniques [22]. THz imaging has been applied to various cancer types, including basal cell carcinoma [23], brain [24], colon [11], and breast [2-7,10,14,21]. For breast cancer in particular, THz spectroscopy has shown distinct differentiation between cancer and healthy tissue at $\mathrm{THz}$ frequencies [4]. As a result, THz shows promise as a margin assessment tool, although some challenges remain such as overlapping properties between fibro-glandular and cancer tissues in some tumor cases.

It should be noted that $\mathrm{THz}$ imaging is limited to providing macroscopic images of tissue and not microscopic ones like pathology. Furthermore, the penetration depth of $\mathrm{THz}$ signal in fresh tissue is limited to few hundred micrometers due to the high absorption of tissue as a medium. For example, the penetration depth at $0.5 \mathrm{THz}$ is $\sim 276 \mu \mathrm{m}$ in cancer, $\sim 360 \mu \mathrm{m}$ in fibro and $\sim 1.142 \mathrm{~mm}$ in fat. It is well known that penetration depth decreases as frequency increases, making $\mathrm{THz}$ suitable for near surface reflection imaging but more limited in transmission in fresh tissue.

A notable challenge in working with freshly excised human breast cancer tumors is that they are hard to obtain without placing the THz imaging system in a surgical setting. Instead we procured specimens of cancer, alone or adjacent to healthy tissues, through the National Disease Research Interchange (NDRI) biobank, in addition to healthy tissue obtained from breast reduction surgeries. To access a greater frequency of samples with lower cost, we have also used animal models such as xenograft mice where cancer cells were injected to grow tumors and transgenic mice where tumors were spontaneously grown. We also developed phantom breast tumor tissues that mimic freshly excised human tumors with a greatly reduced cost.

\section{Terahertz imaging and spectroscopy system}

All experimental results in our research were obtained using the TPS Spectra 3000 terahertz imaging and spectroscopy system, which uses a Ti:Sapphire infrared laser to excite THz antennas fabricated on a low temperature GaAs substrates (Teraview, Ltd., UK) [9]. The system diagram is shown in Fig. 1(a), where the sample under test is positioned in the reflection mode. The $\mathrm{THz}$ signal generated by the antenna is then directed to the sample space using mirrors, with interchangeable modules used for reflection or transmission orientations [21]. The generated THz time domain signal is shown in Fig. 1(b), with a pulse width of $\sim 280 \mathrm{fs}$ for the maximum, $\sim 587 \mathrm{fs}$ for the first minimum, and $\sim 287 \mathrm{fs}$ for the second minimum. Performing Fourier transform on the time domain pulse results in the frequency domain spectrum from 0.1 to $4 \mathrm{THz}$ seen in Fig. 1(c). The overall average THz power of the system is $\sim 1 \mu \mathrm{W}$ 


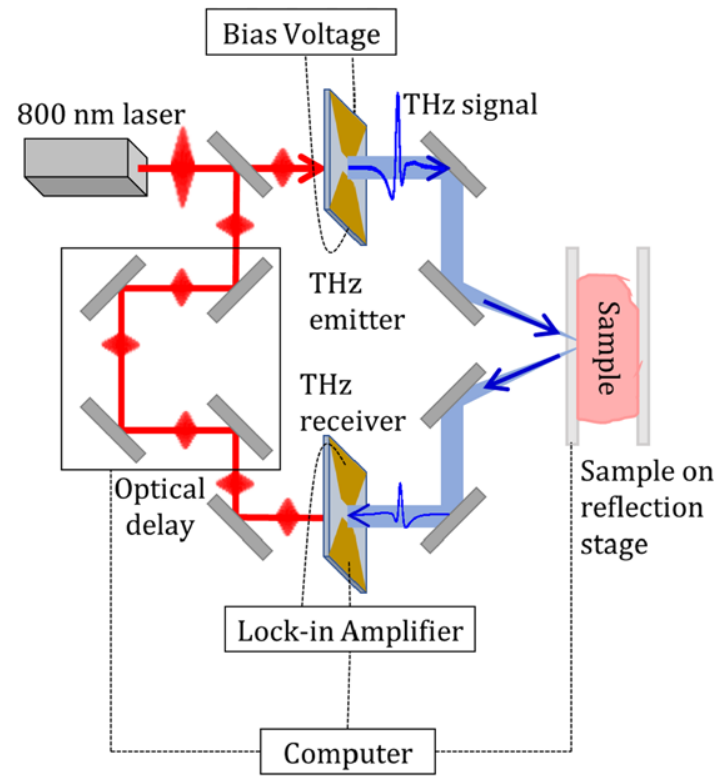

(a)

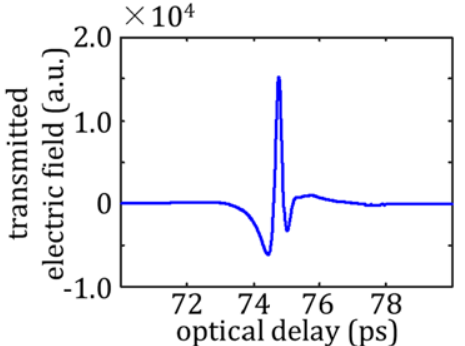

(b)

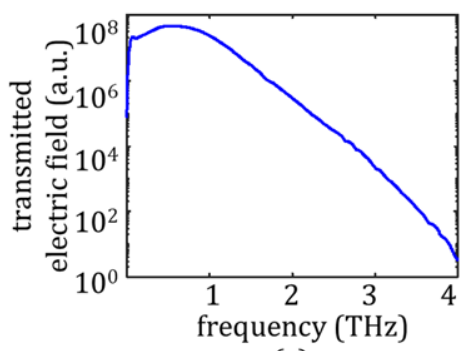

(c)

Fig. 1. (a) Terahertz imaging system diagram for the reflection mode of fresh tumors. (b) Incident time domain THz pulse, and (c) frequency domain signal following Fourier transform [21].

[7]. In addition to the reflection imaging mode, the THz TPS Spectra 3000 system provides scanning in the transmission imaging mode. For biological tissues, where the medium is highly lossy due to the presence of blood and other fluids, the reflection imaging is more suitable compared with transmission imaging [3]. The scanning mechanism of the system uses stepper motors attached to the stage where the tissue is positioned. These motors move with a user-defined step size ranging from $50 \mu \mathrm{m}$ to $500 \mu \mathrm{m}$. The smaller the step size, the higher the resolution of the image, but the longer the imaging time. For a typical breast cancer sample of $1 \mathrm{~cm} \times 1 \mathrm{~cm}$, the image would take $\sim 30-40$ minutes using step size of $200 \mu \mathrm{m}$. The system also provides spectroscopy measurements at room temperature, low temperature down to $4 \mathrm{~K}$ using a cryostat and helium gas, and high temperature up to $523 \mathrm{~K}$ using a heating cell. All our cancer research was conducted at room temperature.

\section{Terahertz spectroscopy}

Characterization of freshly excised human tumor tissue regions to understand their interactions with $\mathrm{THz}$ waves is achieved through spectroscopy measurements. A small piece of the freshly excised tissue is placed between two quartz windows with a spacer of $0.1 \mathrm{~mm}$ thickness inside a liquid sample holder and measured via transmission spectroscopy at a single point at room temperature, as shown in Fig. 2. An average of $\sim 1800$ signals is taken to reduce the random noise of the system, and nitrogen gas purging is used to remove any effect from water vapor in the measurement [4]. An inversion algorithm minimizes the amplitude and phase error between the measured data and a theoretical model to extract the refractive index $(n)$ and absorption coefficient $(\alpha)$ in the range from $0.1 \mathrm{THz}$ to $4 \mathrm{THz}$. The spectroscopy model in all of our work is based on the transmitted electric field through the threelayer system (quartz-tissue-quartz) of the liquid sample holder shown in Fig. 2(c). However, systems of 


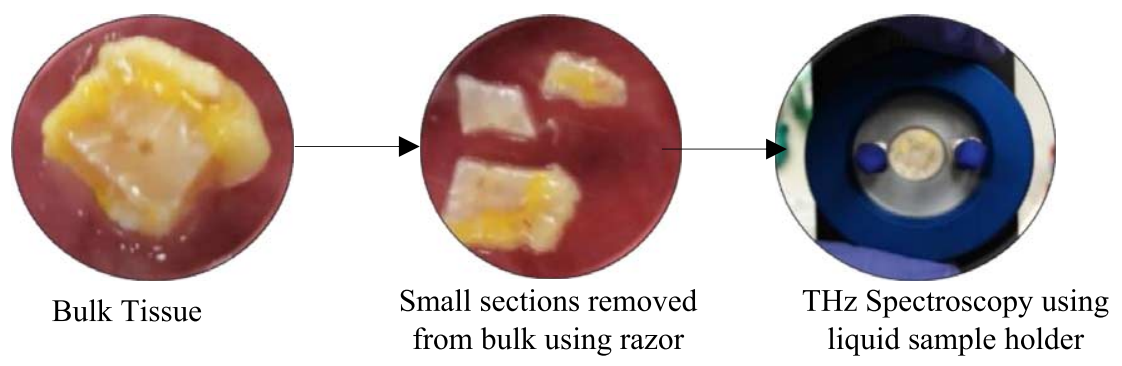

(a)

(b)

(c)

Fig. 2. Sample preparation for THz spectroscopy. (a) Photograph of the whole tissue. (b) Photograph of pieces cut from the tissue. (c) Placing the small piece of the tissue in the liquid sample holder for spectroscopy procedure.

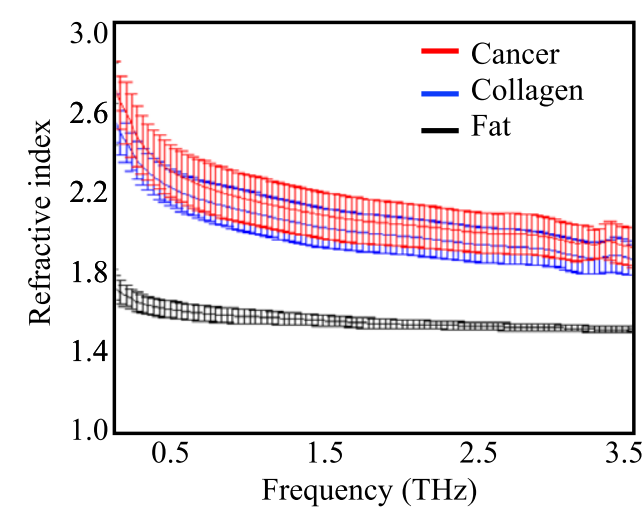

(a)

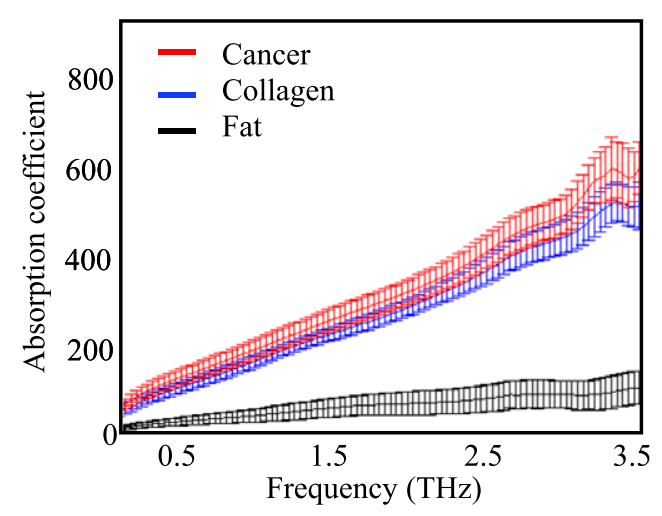

(b)

Fig. 3. The mean spectroscopy data for all fresh human cancer and healthy breast tissues. (a) The transmission refractive indices for cancer, collagen, and fat. (b) The transmission absorption coefficient for cancer, collagen, and fat. The transmission spectroscopic data in (a) and (b) is average over 10 individual points from each tissue type [4].

different layers or in reflection mode require alternative considerations for model accuracy $[3,4]$. The transmission spectroscopy results of freshly excised human breast tumors of infiltrating ductal carcinoma (IDC), fibro-glandular, and fatty tissues are shown in Fig. 3. The results demonstrate a significant differentiation between cancer and fat and less clear differentiation between cancer and fibro-glandular tissue, which is an ongoing challenge [4].

\section{Terahertz imaging of freshly excised tumors}

For each fresh tissue sample, we produce three images: the THz fresh image, THz FFPE image, and pathology image. The process is summarized in [21]. Core THz system imaging utilizes modules for transmission or reflection orientations [3]. Tissues are mounted on polystyrene slides for transmission or polystyrene windows for reflection due to low absorption $[3,6]$. THz measurements are taken by scanning the sample and comparing the reflected or transmitted signal at each point against a reference, generally a gold mirror or a known material (reflection) or an empty sample holder (transmission). Normalizing the measured signal with a carefully selected reference is vital to compensate for any phase changes [3]. From there, the THz image can be generated by taking the time domain peak or single frequency 


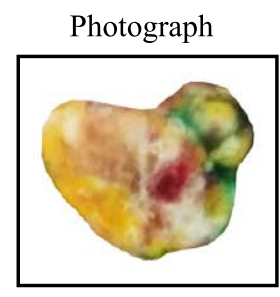

(a)

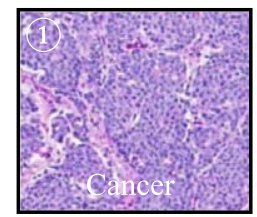

(e)

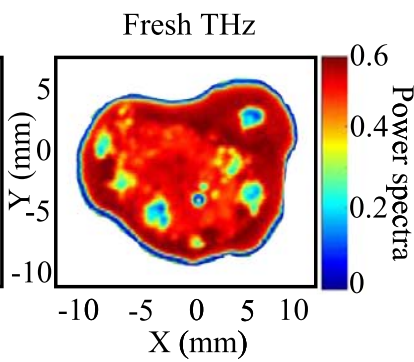

(b)

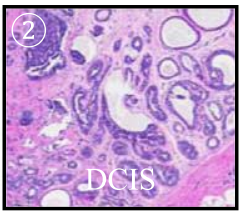

(f)

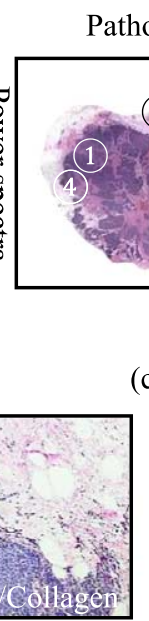

(g)

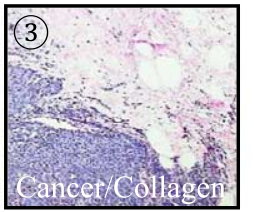

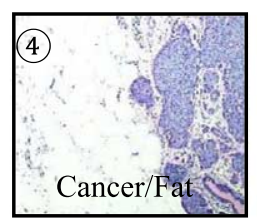

(h)

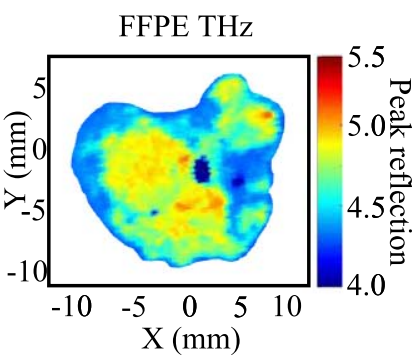

(d)

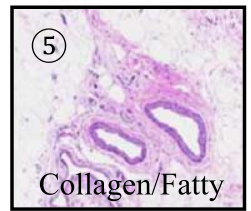

(i)

Fig. 4. THz images of human breast cancer tissue. (a) The photograph of fresh tissue. (b) The frequency domain THz image of fresh tissue represented in spectral power. (c) The low power pathology image. (d) The time domain THz image of FFPE block tissue. (e) The high power pathology image of cancer region marked as (1) in (c), (f). The high power pathology image of DCIS region marked as (2) in (c), (g). The high power pathology image of cancer-collagen region marked as (3) in (c), (h). The high power pathology image of cancer-fat region marked as (4) in (c), (i). The high power pathology image of collagen-fatty region marked as (5) in (c).

magnitude at each point [2], deconvolving the reference signal [7], integrating the frequency domain spectral power [2,4,10,21], or implementing statistical signal processing for cancer classification [2] and [10].

We investigated imaging three types of freshly excised tumors. The first was human breast cancer obtained from surgery through biobanks. Most specimens were obtained from NDRI as direct lumpectomy or mastectomy surgical tissue placed in Dulbecco's Modified Eagle Medium (DMEM) to maintain tissue health with penicillin streptomycin (PenStrep) to serve as an antibiotic. Specimens were shipped overnight on wet ice, and $\mathrm{THz}$ imaging was performed within 24 hours of surgery. This is currently the fastest option to obtain freshly excised human tumors without placing the $\mathrm{THz}$ system directly into a surgical setting [4].

An example human cancer specimen is presented in Fig. 4, showing a heterogeneous tissue that includes adjacent invasive mammary carcinoma (IMC), ductal carcinoma in situ (DCIS), fibrous, and fatty tissues. This sample was obtained from a 69 years old female who underwent a left breast mastectomy procedure. In this case, we focused on detecting the cancer on the margins using $\mathrm{THz}$ imaging. The dark purple color region in Fig. 4(c) represents the cancer shown as light red in the fresh $\mathrm{THz}$ image in Fig. 4(b). The blue color regions in Fig. 4(b) represents the presence of fat in the tissue while the dark red color represents the healthy collagen as can be seen in the pathology image in Fig. 4(c) in white and light purple colors, respectively. The THz image of the FFPE tissue block in Fig. 4(d) shows significant differentiation between the cancerous and non-cancerous regions. The yellow-reddish color region indicates cancer, the turquoise blue color indicates collagen, and the blue color indicates fat. Furthermore, DCIS shown in the pathology image of Fig. 4(c) (marked by as (2)), is represented in yellow color in Fig. 4(d), similar to cancer. However, the DCIS region in the tissue could not be differentiated from cancer in the fresh THz image of Fig. 4(b). This could be due to a possible change in the surface of the fresh tissue during the histopathology process, resulting in different features in these images. 


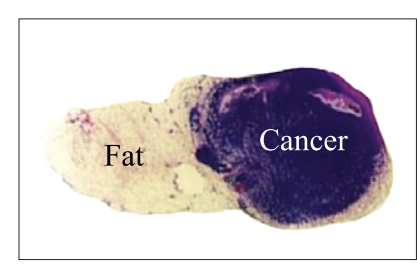

(a)

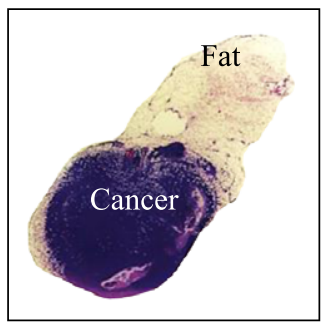

(c)

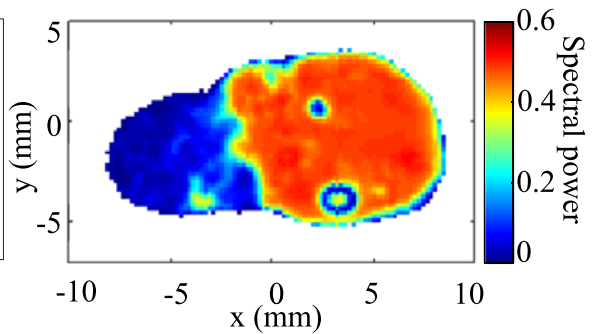

(b)

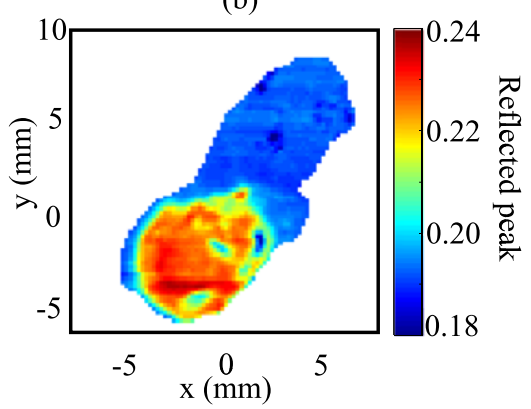

(d)

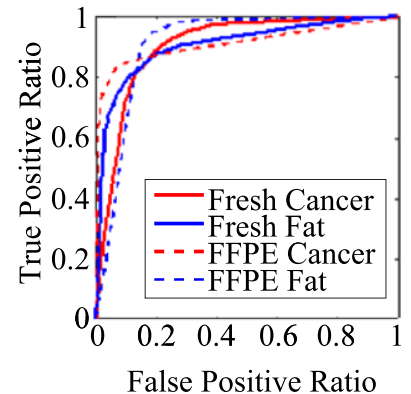

(e)

Fig. 5. THz images of xenograft mice tumor sample 2. (a) Pathology image, (b) THz image of freshly excised tissue, (c) pathology image (same as in (a)), (d) THz image of FFPE block tissue, and (e) the statistical classification [2].

The other two types of fresh tumor were obtained from xenograft [2] and transgenic tumors [21]. Xenograft tumors are grown via cell injection of murine-derived E0771 breast adenocarcinoma cells. For our work, tumors were grown in the fat deposits of C57BL/6 black laboratory mice from the Jackson Laboratory maintained on a high fat diet (D12492 from Research Diets, Inc.) and excised once they reached $1 \mathrm{~cm}$ in diameter [1]. Excised tumors were transferred in phosphate-buffered saline (PBS) to our $\mathrm{THz}$ system for imaging immediately after excision. Histopathology assessment was then performed by Oklahoma Animal Disease Diagnostic Laboratory (OADDL) followed by FFPE block imaging. Results from one xenograft mouse in Fig. 5 show significant differentiation between cancer and fat. Additional work mapped the pathology assessment of the tissue for statistical comparison to the regions of tissue in the THz image, providing accuracy curves shown in Fig. 5(e) [2]. It should be noted that these mice tumors do not contain fibro-glandular tissues due to the lack of natural fibrous tissue production around the tumor site, and therefore represent an ideal case rather than a realistic one.

Transgenic mice tumors occur naturally due to selective breeding to eliminate a cancer suppressor gene [21]. As such they represent spontaneous tumor growth with associated structures and vasculature, serving as better representations of human tissue than xenograft tumors. The transgenic tumors were excised from MMTV-PyMT mice obtained from the Jackson Laboratory, and the THz imaging results were reported in [21]. The sample in Fig. 6 demonstrates the significant difference in the structure, complexity, and heterogeneity between transgenic and xenograft tumors shown in Fig. 5. While tumors excised from transgenic mice are more complex in structure, samples in practice mainly contain cancer with little fibro and or fatty tissues due to healthy tissue degradation during tumor growth [21]. As such they were not as useful for developing clear differentiation metrics between cancer and healthy tissue. 


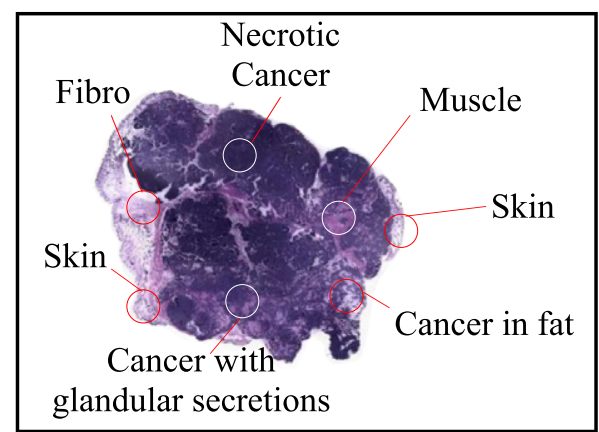

(a)

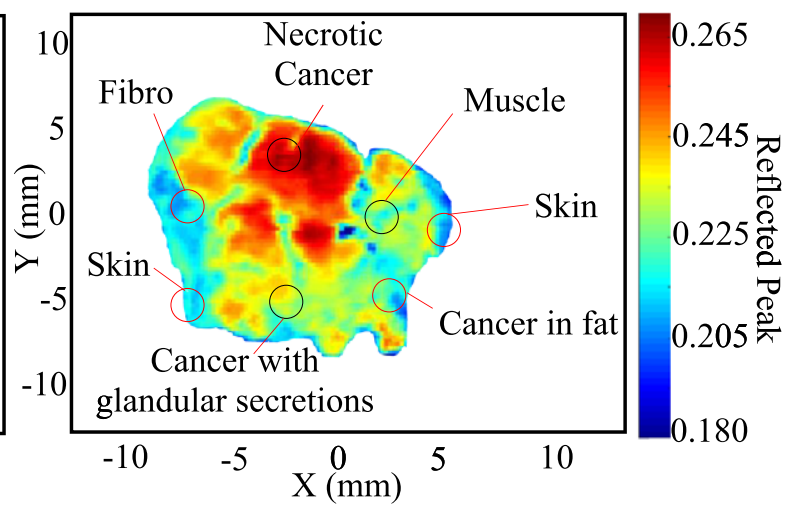

(b)

Fig. 6. Transgenic mice images. (a) H\&E stained pathology image. (b) THz image of FFPE block tissue [21].

\section{Phantom breast tumors}

Since freshly excised breast tumors are expensive and difficult to obtain in large quantities, we developed phantom breast tumors to establish initial imaging methodology and for testing contrast agents [5]. These phantom materials contain a TX151 solidifying powder base with varying amounts of water, oil, and surfactant to adjust the THz properties to align with that of fresh cancer and fibro-glandular tissues, verified using THz spectroscopy [5]. Phantoms allow significant control over the experimental setup and the random organization of cancer and healthy tissue. They also serve as a cost-effective means for developing new imaging methodologies and for examining the effects of possible toxic contrast agents, such as nanoparticles, on the effective reflection from tissue [5].

Our first version of phantom cancer (IDC) was composed of $66 \%$ water, $12 \%$ oil, $6 \%$ surfactant, $14 \%$ TX151, and $2 \%$ agar. While, phantom fibro was composed of $56 \%$ water, $18 \%$ oil, $10 \%$ surfactant, $14 \%$ TX151, and 2\% agar [5]. The phantom fatty tissue was composed of butter (solid oil-water emulsion). However, due to the presence of air bubbles and the soft texture of the fatty phantom tissues, we optimized the design as reported in [19]. The phantom model tumors that contained cancer and fibro with and without onion-like carbon (OLC) as a contrast agent are presented in Fig. 7 and reported in [5]. It was concluded that OLC represented a good contract agent to enhance the differentiation between cancerous and fibro phantom breast tissues in $\mathrm{THz}$ imaging. While a $1 \%$ OLC by weight against the tissue phantom showed little effect, a higher presence of OLC drastically changed the $\mathrm{THz}$ reflection. More investigation is needed to define how much OLC is practical in a surgical setting. Additionally, the toxicity of OLC requires further research in the future. Current research to develop three-dimensional phantom models with heterogeneous structures aims to mimic realistic human breast cancer tumors.

\section{Conclusions}

Terahertz imaging and spectroscopy technology has shown potential in detecting cancer in excised breast tumors without the use of contrast agent. An advantage of $\mathrm{THz}$ imaging that it provides a whole slide image of the specimen. The results demonstrated a strong differentiation between cancerous and non-cancerous regions. In THz imaging of dehydrated tissues, the contrast between the cancer and connective healthy tissues (fibroglandular) is significant and closely aligned with pathology. THz images of 


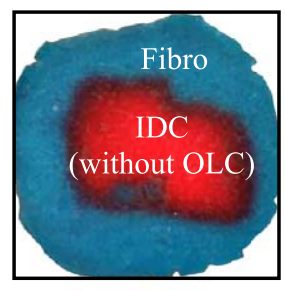

(a)

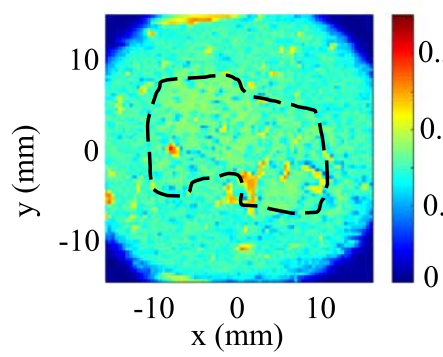

(d)

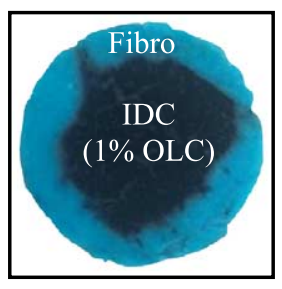

(b)

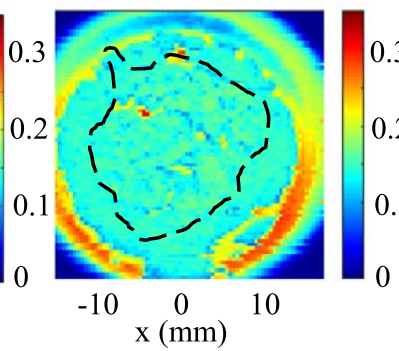

(e)

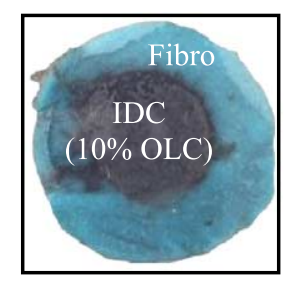

(c)

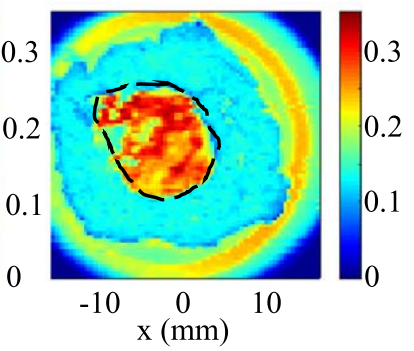

(f)

Fig. 7. THz reflection imaging of combined phantoms shown in photographs for (a) photo of IDC phantom with no onion-like carbon (OLC), (b) photo of IDC phantom with $1 \%$ of $100 \mathrm{~nm}$ OLC, and (c) photo of IDC phantom with $10 \%$ of $100 \mathrm{~nm}$ OLC. THz images in (d)-(f) show the resulting THz reflection images for (d) no OLC in IDC, (e) 1\% OLC in IDC, and (f) $10 \%$ OLC in IDC [5].

freshly excised tissues show reasonably good contrast between different tissue regions as well, though direct comparison with pathology remains a challenge due to changes to the tissue during the histopathology process. A strong pathology correlation and development of the intraoperative technique continues to be the focus of future work.

\section{Acknowledgements}

This work was funded in part by the National Institutes of Health Award No. R15CA208798 and by the National Science Foundation (NSF) Award No. 1408007. We acknowledge the use of human tissues procured by the National Disease Research Interchange (NDRI) with support from NIH Grant No. U42OD11158. All animal research was in compliance with the guidelines outlined in the Guide for the Care and Use of Laboratory Animals. Animal tumors were grown and excised in Dr. N. Rajaram's lab in the Biomedical Engineering Department at the University of Arkansas.

\section{Conflict of interest}

The authors have no conflict of interest to report.

\section{References}

[1] S.K. Boi et al., Obesity alters immune and metabolic profiles: New insight from obese-resistant mice on high-fat diet, Obesity 24 (2016), 2140-2149. doi:10.1002/oby.21620. 
[2] T. Bowman, T. Chavez, K. Khan, J. Wu, A. Chakraborty, N. Rajaram, K. Bailey and M. El-Shenawee, Pulsed terahertz imaging of breast cancer in freshly excised murine tumors, J. Biomed. Opt. 23(2) (2018). doi:10.1117/1.JBO.23.2.026004.

[3] T. Bowman, M. El-Shenawee and L.K. Campbell, Terahertz transmission vs reflection imaging and model-based characterization for excised breast carcinomas, Biomed. Opt. Express 7(9) (2016). doi:10.1364/BOE.7.003756.

[4] T. Bowman, N. Vohra, K. Bailey and M. El-Shenawee, Terahertz tomographic imaging of freshly excised human breast tissues, J. Med. Imag. 6(2) (2019), 023501.

[5] T. Bowman, A. Walter, O. Shenderova, N. Nunn, G. McGuire and M. El-Shenawee, A phantom study of terahertz spectroscopy and imaging of micro- and nano-diamonds and nano-onions as contrast agents for breast cancer, Biomed. Phys. Eng. Express 3(5) (2017), 55001. doi:10.1088/2057-1976/aa87c2.

[6] T. Bowman, Y. Wu, J. Gauch, L.K. Campbell and M. El-Shenawee, Terahertz imaging of three-dimensional dehydrated breast cancer tumors, J. Infrared, Millimeter, Terahertz Waves 38(6) (2017), 766-786. doi:10.1007/s10762-017-0377-y.

[7] T.C. Bowman, M. El-Shenawee and L.K. Campbell, Terahertz imaging of excised breast tumor tissue on paraffin sections, IEEE Trans. Antennas Propag. 63(5) (2015), 2088-2097. doi:10.1109/TAP.2015.2406893.

[8] F. Bray, J. Ferlay, I. Soerjomataram, R.L. Siegel, L.A. Torre and A. Jemal, Global cancer statistics 2018: GLOBOCAN estimates of incidence and mortality worldwide for 36 cancers in 185 countries, Cancer J. Clin. 68(6) (2018), 394-424. doi:10.3322/caac.21492.

[9] N.M. Burford and M. El-Shenawee, Review of terahertz photoconductive antenna technology, Opt. Eng. 56(1) (2017), 010901. doi:10.1117/1.OE.56.1.010901.

[10] T. Chavez, T. Bowman, J. Wu, K. Bailey and M. El-Shenawee, Assessment of terahertz imaging for excised breast cancer tumors with image morphing, J. Infrared Milli. Terahz, Waves 39(12) (2018), 1283-1302. doi:10.1007/s10762-0180529-8.

[11] P. Doradla, K. Alavi, C. Joseph and R. Giles, Detection of colon cancer by continuous-wave terahertz polarization imaging technique, J. Biomed. Opt. 18 (2013), 90504. doi:10.1117/1.JBO.18.9.090504.

[12] S. Fan, Y. He, B.S. Ung and E. Pickwell-MacPherson, The growth of biomedical terahertz research, J. Phys. D. Appl. Phys. 47(37) (2014), 374009. doi:10.1088/0022-3727/47/37/374009.

[13] S. Glück and T. Mamounas, Improving outcomes in early-stage breast cancer, Oncology 24(11) (2010), Suppl. 4, 1-15.

[14] M.R. Grootendorst, A.J. Fitzgerald, S.G. Brouwer de Koning, A. Santaolalla, A. Portieri, M. Van Hemelrijck, M.R. Young, J. Owen, M. Cariati, M. Pepper, V.P. Wallace, S.E. Pinder and A. Purushotham, Use of a handheld terahertz pulsed imaging device to differentiate benign and malignant breast tissue, Biomedical Optics Express 8(6) (2017), 2932-2945. doi:10. 1364/BOE.8.002932.

[15] L. Jacobs, Positive margins: The challenge continues for breast surgeons, Ann. Surg. Oncol. 15(5) (2008), $1271-1272$. doi:10.1245/s10434-007-9766-0.

[16] V.S. Klimberg, C. Boneti, L.L. Adkins, M. Smith, E. Siegel, V. Zharov, S. Ferguson, R. Henry-Tillman, B. Badgwell and S. Korourian, Feasibility of percutaneous excision followed by ablation for local control in breast cancer, Ann. Surg. Oncol. 18(11) (2011), 3079-3087. doi:10.1245/s10434-011-2002-y.

[17] V.S. Klimberg, J. Kepple, G. Shafirstein, L. Adkins, R. Henry-Tillman, E. Youssef, J. Brito, L. Talley and S. Korourian, eRFA: Excision followed by RFA - A new technique to improve local control in breast cancer, Ann. Surg. Oncol. 13(11) (2006), 1422-1433. doi:10.1245/s10434-006-9151-4.

[18] M.S. Moran, S.J. Schnitt, A.E. Giuliano, J.R. Harris, S.A. Khan, J. Horton, S. Klimberg, M. Chavez-MacGregor, G. Freedman, N. Houssami, P.L. Johnson and M. Morrow, Society of Surgical Oncology-American Society for Radiation Oncology Consensus guideline on margins for breast-conserving surgery with whole-breast irradiation in stages I and II invasive breast cancer, Int. J. Radiat. Oncol. 88(3) (2014), 553-564. doi:10.1016/j.ijrobp.2013.11.012.

[19] C. Oldfield, T. Bowman and M. El-Shenawee, Development of tunable breast tissue phantoms for terahertz imaging, in: Proc. of IEEE-APS/URSI 2018, Boston, MA, 8-13 July, 2018.

[20] R.G. Pleijhuis, M. Graafland, J. de Vries, J. Bart, J.S. de Jong and G.M. van Dam, Obtaining adequate surgical margins in breast-conserving therapy for patients with early-stage breast cancer: Current modalities and future directions, Ann. Surg. Oncol. 16(10) (2009), 2717-2730. doi:10.1245/s10434-009-0609-z.

[21] N. Vohra, T. Bowman, P.M. Diaz, N. Rajaram, K. Bailey and M. El-Shenawee, Pulsed terahertz reflection imaging of tumors in a spontaneous model of breast cancer, Biomedical Physics and Engineering Express 4(6) (2018), 065025. doi:10.1088/2057-1976/aae699.

[22] G.J. Wilmink and J.E. Grundt, Invited review article: Current state of research on biological effects of terahertz radiation, J. Infrared, Millimeter, Terahertz Waves 32(10) (2011), 1074-1122. doi:10.1007/s10762-011-9794-5.

[23] R.M. Woodward, V.P. Wallace, R.J. Pye, B.E. Cole, D.D. Arnone, E.H. Lin and M. Pepper, Terahertz pulse imaging of ex vivo basal cell carcinoma, J. Invest. Dermatol. 120(1) (2003), 72-78. doi:10.1046/j.1523-1747.2003.12013.x.

[24] S. Yamaguchi, Y. Fukushi, O. Kubota, T. Itsuji, T. Ouchi and S. Yamamoto, Brain tumor imaging of rat fresh tissue using terahertz spectroscopy, Sci. Rep. 6 (2016), 1-6. doi:10.1038/s41598-016-0001-8. 